\title{
Síndrome de Cushing durante el embarazo: manifestaciones cutáneas secundarias a un tumor suprarrenal
}

\author{
Montserrat Molgó $N_{\dot{3}}{ }^{1}$, David Mayerson $B .^{2}$, Pedro Jaramillo $Z^{1}{ }^{1}$, Julio Parra $C .^{4}$, \\ Sergio González B. ${ }^{3}$
}

${ }^{1}$ Departamento de Dermatología, ${ }^{2}$ Departamento de Ginecología y Obstetricia, ${ }^{3}$ Departamento de Anatomía Patológica, Escuela de Medicina, Pontificia Universidad Católica de Chile. ${ }^{4}$ Unidad de Teledermatología, Centro de Salud Familiar San Fabián, Servicio de Salud Ñuble. Chile.

Financiamiento: Esta publicación fue completamente financiada por los Departamentos de Dermatología y GinecologíaObstetricia de la Pontificia Universidad Católica de Chile.

\section{RESUMEN}

El síndrome de Cushing secundario a tumor suprarrenal es una patología infrecuente en el embarazo. Su presencia se traduce en un aumento significativo de la morbimortalidad materna y fetal. Muchas de sus características clínicas son enmascaradas por los cambios propios de una gestación fisiológica, lo que dificulta el diagnóstico. En este contexto las manifestaciones dermatológicas pueden ser de gran utilidad en la sospecha clínica precoz. Se presenta el caso de una paciente de 26 años de edad que en el segundo trimestre de embarazo inicia un cuadro caracterizado por acné inflamatorio severo e hirsutismo. La gestación se interrumpe a las 35 semanas por síndrome hipertensivo del embarazo y rotura prematura de membranas. Durante el puerperio consulta al dermatólogo presentando aún las lesiones descritas, además de estrías violáceas gruesas en abdomen, facie de luna, obesidad centrípeta y máculas purpúricas en zonas de presión. Se diagnóstica síndrome de Cushing. El estudio confirmatorio y etiológico dirigido evidenció un adenoma de corteza suparrenal. El diagnóstico de laboratorio es difícil debido a los cambios físicos y de laboratorio habituales del embarazo que pueden mimetizar los hallazgos propios de la enfermedad. Los hallazgos dermatológicos pueden ser de gran valor para un diagnóstico y tratamiento precoz. Las lesiones dermatológicas secundarias al hipercortisolismo fueron de difícil manejo.

PALABRAS CLAVE: Embarazo, hirsutismo, acné, síndrome de Cushing, adenoma adrenal

\section{SUMMARY}

Cushing's syndrome secondary to adrenal tumor is a rare pathology in pregnancy. Its presence results in a significant increase in maternal and fetal morbimortality. Many of its clinical features are masked by the typical changes of a physiological pregnancy, which make difficult the diagnosis. Indeed, skin manifestations may be useful in early clinical suspicion. We report a case of a 26 year old in the second trimester with severe inflammatory acne and hirsutism. The pregnancy is interrupted at 35 weeks for pregnancy-induced hypertension syndrome and premature rupture ovular membranes. During the puerperium, patient consulted a dermatologist presenting still the injuries described, as well as thick purple striae throughout the abdomen, moon face, truncal obesity and purpuric macules in pressure zones. Cushing's syndrome is diagnosed. The study showed a cortex suprarenal adenoma. Laboratory diagnosis is difficult because the pregnancy physical changes and routine laboratory findings that can mimic the disease themselves. Dermatological 
findings may be valuable for diagnosis and early treatment. The skin lesions secondary to hypercortisolism were difficult to manage.

\section{KEY WORDS: Pregnancy, hirsutism, acne, Cushing syndrome, adrenal adenoma}

\section{INTRODUCCIÓN}

El síndrome de Cushing secundario a tumor suprarrenal produce un estado de infertilidad asociado al exceso de glucocorticoides, por lo que resulta una condición infrecuente durante el embarazo, existiendo a la fecha cerca de 60 casos reportados $(1,2)$. La causa más frecuente del hipercortisolismo en el embarazo es el síndrome de Cushing ACTHindependiente (1-8).

Su presencia se traduce en un aumento significativo de la morbimortalidad materna y fetal. La mortalidad materna puede llegar al $4,5 \%$ de los casos, asociándose a hipertensión y diabetes (5). La mortalidad fetal es alta, secundaria a abortos espontáneos, mortinatos e infección post natal, oscilando entre 1,5 al $20 \%$ de los embarazos. La restricción del crecimiento intrauterino ocurre en un $15 \%(4,5,9)$. Esto está agravado, ya que en el $60 \%$ de los embarazos terminan en parto prematuro (5). Estas cifras hacen necesario su diagnóstico precoz.

Muchas de sus características clínicas son enmascaradas por los cambios propios de una gestación fisiológica o patologías propias del embarazo, como la hipertensión, diabetes gestacional, estrías abdominales y edema, lo que dificulta el diagnóstico. El embarazo afecta dramáticamente el eje hipotálamo-hipófisis-suprarrenal (7), por lo que las mediciones hormonales son difíciles de interpretar. En este contexto el cuadro clínico dermatológico es de gran utilidad en la sospecha clínica (1-3).

Presentamos el caso de una paciente con manifestaciones dermatológicas clásicas de hipercortisolismo durante la segunda mitad del embarazo, cuyo diagnóstico sindromático y etiológico solo fue posible establecer en el puerperio.

\section{Caso clínico}

Se presenta el caso de una paciente de 26 años, sin antecedentes mórbidos a destacar. Durante el segundo trimestre de embarazo inicia un cuadro caracterizado por acné inflamatorio severo, hirsutismo y síndrome hipertensivo del embarazo desde las 28 semanas. Se maneja como preeclampsia. A las 35 semanas presenta una rotura prematura de membranas, por lo que se decide su interrupción.

Se deriva a dermatólogo a los 15 días de puerperio, presentando al examen físico pápulo-pústulas monomorfas en cara, pecho y espalda, con importante aumento del vello en región mandibular y espalda, con presencia de pelo terminal, facie de luna llena, relleno supraclavicular, máculas purpúricas en zonas de manguito de presión y abdomen abultado con estrías violáceas gruesas (Figura 1)

Con la sospecha clínica de síndrome de Cushing, se inició el estudio de laboratorio, destacando cortisol basal de 27,8 ug/dL (VN: 6,4-15 ug/dL) y post ACTH 27,9 ug/dL a los 30 minutos (VN: >20 $\mathrm{ug} / \mathrm{dL}$ ), ACTH $<5 \mathrm{pg} / \mathrm{mL}$ (VN: $10-60 \mathrm{pg} / \mathrm{mL}$ ), cortisol urinario $588 \mathrm{ug} / \mathrm{dL}$ (VN: 20-90 ug/dL). Presentaba además colesterol total de $311 \mathrm{mg} / \mathrm{dL}$ y LDL 214 $\mathrm{mg} / \mathrm{dL}, 17-\mathrm{OH}$ corticoesteroides $18,6 \mathrm{mg} / 24 \mathrm{hrs}$ (VN: $2-8 \mathrm{mg} / 24 \mathrm{hrs}$ ) y cetoesteroides $56,3 \mathrm{mg} / 24 \mathrm{hrs}$ (VN: $4-16 \mathrm{mg} / 24 \mathrm{hrs}$ ), testosterona total, DHEAS, SHBG, hemograma, pruebas tiroideas y electrolitos plasmáticos en rangos normales.

El estudio por imágenes con resonancia nuclear magnética de abdomen mostró tumor cortical suprarrenal izquierdo, por lo que se realizó adrenalectomía laparoscópica, cuyo estudio histopatológico fue compatible con adenoma de corteza suprarrenal (Figura 2).

El manejo de las lesiones cutáneas fue difícil: el acné no respondió a tratamientos habituales, requiriendo uso de antibióticos, terapia fotodinámica y Dapsona, que al cabo de 2 meses debió suspenderse por anemia hemolítica. Las estrías se trataron con ácido retinoico tópico y luz pulsada intensa.

\section{DISCUSIÓN}

El síndrome de Cushing es una patología infrecuente en el embarazo, ya que la mayoría de las pacientes presentan infertilidad (1). El diagnóstico de hipercortisolismo es difícil porque muchos de los síntomas se presentan en formas leves, o en formas graves que se interpretan como complicaciones del embarazo (4-5). Dentro de ellas, la hipertensión arterial y las alteraciones en el metabolismo de los hidratos de carbono ocurren hasta en un $80 \%$ y $25 \%$, respectivamente (1-7). Algunas complicaciones menos frecuentes son la preclampsia, eclampsia, tromboembolismo pulmonar, edema pulmonar y la falla cardiaca congestiva (4-6). Otras condiciones asociadas al hipercortisolismo son la infección o deshicencia de las heridas operatorias (hasta en un $40 \%$ ) en aquellas pacientes sometidas a operación cesárea (4). Por lo anterior es importante el diagnóstico precoz que permita un seguimiento y manejo adecuado. Sin embargo el diagnóstico se hace difícil debido a los cambios físicos y de laboratorio propios del embarazo, que pueden enmascarar los hallazgos de la enfermedad. 


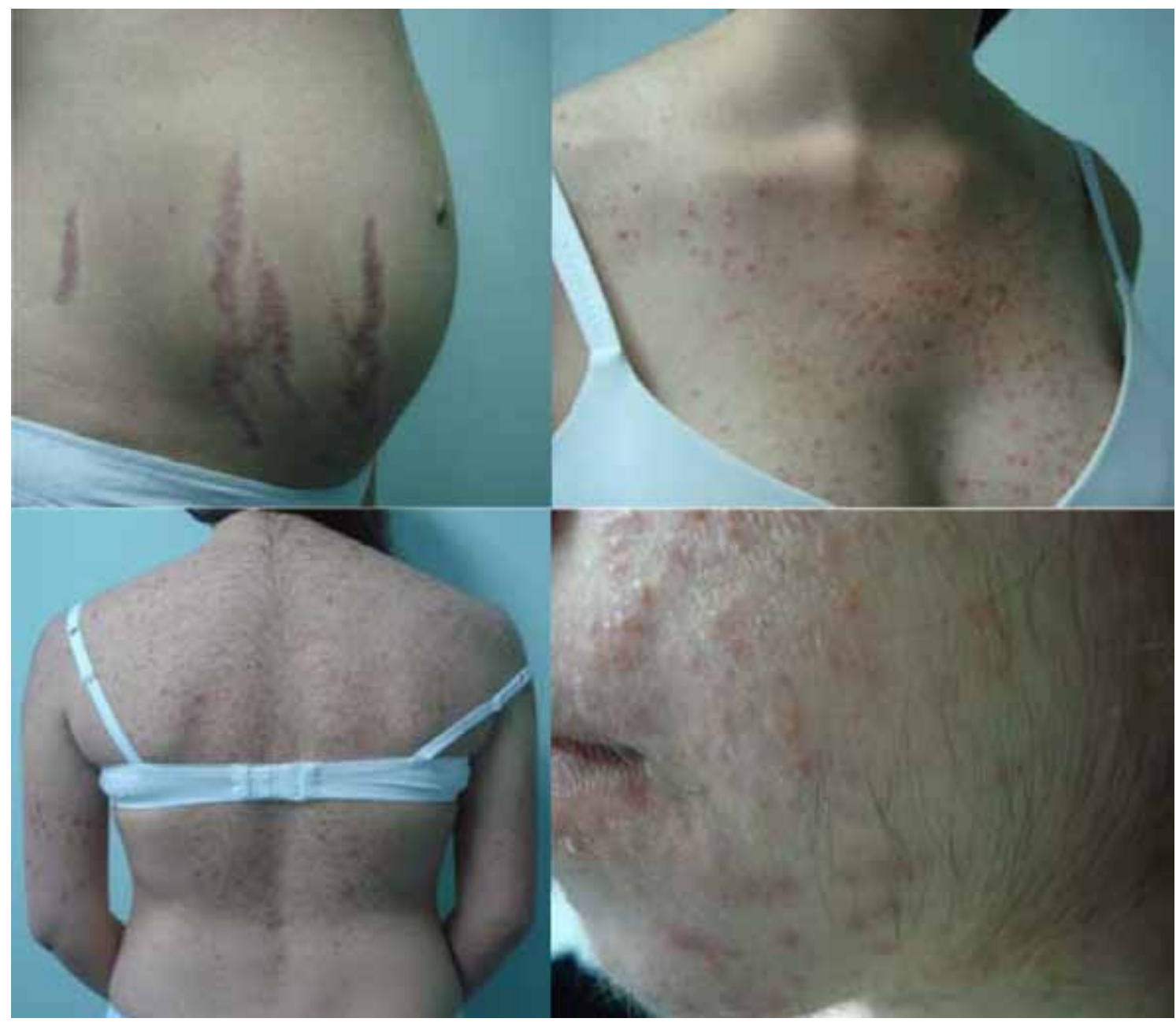

Figura 1. Abdomen gravídico con estrías violáceas gruesas, pápulo-pústulas monomorfas en pecho, espalda e hirsutismo en cara.

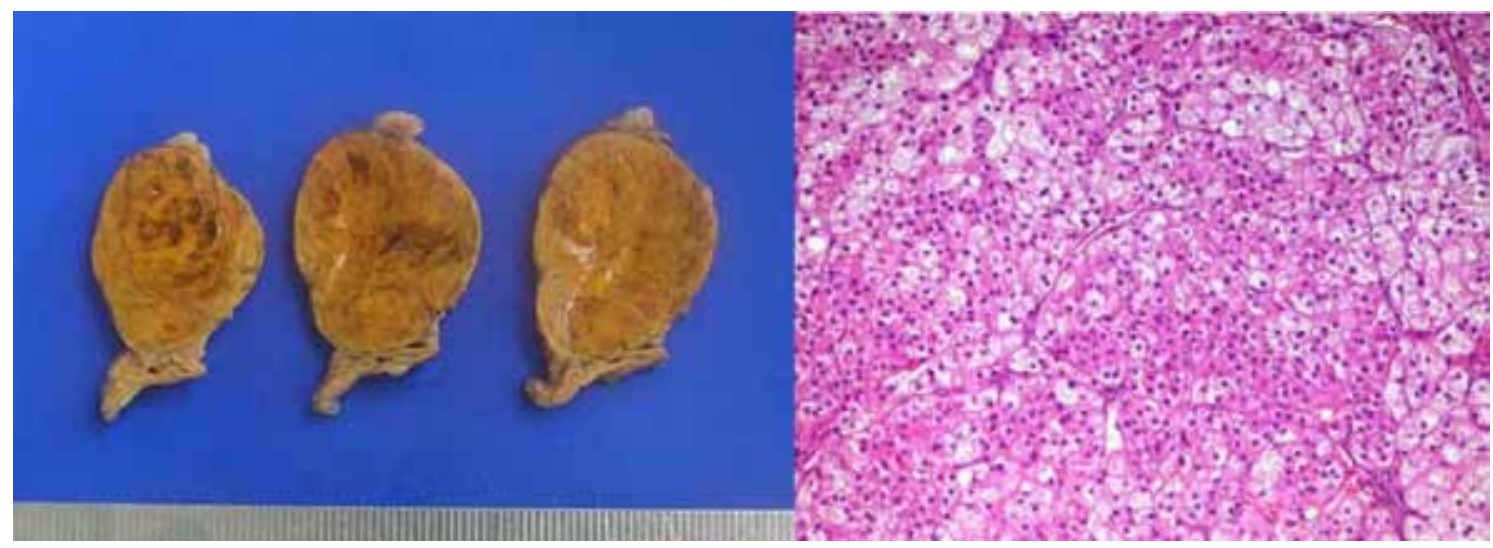

Figura 2. Adenoma de corteza suprarrenal macroscópico y a la microscopía de luz clara. 
En el examen físico, el aumento de peso en el hipercortisolismo es principalmente centrípeto, mientras que en una embarazada normal es habitualmente más uniforme. Otras claves diagnósticas son las manifestaciones cutáneas, entre las que están las estrías violáceas gruesas y la fácil generación de equimosis, seguidas por acné, hirsutismo, alopecia y la mala cicatrización (1-5). En este caso, la paciente inicia un cuadro de acné inflamatorio severo, hirsutismo, estrías violáceas gruesas en abdomen y disposición centrípeta de la masa grasa.

El estudio y diagnóstico del síndrome de Cushing durante el embarazo se dificulta debido a las alteraciones fisiológicas en el eje hipotálamo-hipófisis-suprarrenal, caracterizadas por aumento de la hormona adrenocorticotropa (ACTH), el cortisol total, cortisol libre y la globulina fijadora de cortisol (CBG). En una gestación fisiológica la excreción de cortisol urinario y salival puede presentar un incremento de hasta tres veces el valor normal, lo que implica que títulos elevados en mediciones basales no traducen necesariamente patología $(4,7,8,10)$. En este caso ante la sospecha clínica de síndrome de Cushing, el estudio se inició con un cortisol basal que resultó elevado. Sin embargo, el diagnóstico fue posible dada su invariabilidad frente a la estimulación con ACTH, que es el test más adecuado para su diagnóstico durante el curso de la gestación y puerperio precoz. Un test alternativo es la prueba de supresión de corticoides con dexametasona, que tiene la desventaja de no suprimir la ACTH placentaria (10).

El estudio por imágenes de la paciente demostró un adenoma de corteza suprarrenal, que es la etiología más frecuente en este contexto $(41,1 \%)(1-3)$.

El diagnóstico del síndrome de Cushing en esta oportunidad se hizo en el puerperio. Si el diagnóstico se realiza durante el embarazo, el tratamiento es complejo, existiendo varias alternativas terapéuticas. Estas dependen de la edad gestacional y la gravedad del caso. La suspensión del tratamiento es de alto riesgo. En pacientes con estadíos leves podría plantearse una conducta expectante (11). Si hay hipertensión deben usarse hipotensores. La dieta y la insulina deben usarse si hay diabetes gestacional. La cirugía, abierta o laparoscópica (4) debe realizarse en el puerperio $(4,5)$, aunque existen reportes de adrenalectomía unilateral durante el embarazo mediante laparoscopía retroperitoneal $(4,12)$. En espera de la cirugía, o cuando está contraindicada, se ha usado Metyrapone, con buena supresión de cortisol, sin efectos descritos en el feto, en dosis de 2,5 a $3 \mathrm{~g}$ día(13-15). Existen otras alternativas, aunque con probables efectos teratogénicos (16-19).

\section{CONCLUSIÓN}

El síndrome de Cushing es una entidad rara durante el embarazo, con poco más de 150 casos reportados en la literatura. La etiología más frecuente es el adenoma suprarrenal. El diagnóstico, al ser muy difícil, se basa fundamentalmente en los hallazgos al examen físico, especialmente a las lesiones cutáneas de aparición aguda, secundarias al hiperandrogenismo y a la aparición de síntomas como hipertensión y trastornos en el metabolismo de los hidratos de carbono. Estas manifestaciones dermatológicas pueden orientar la sospecha precoz de esta patología. Para su diagnóstico debe evaluarse los cambios hormonales que suceden durante una gestación fisiológica. El test más útil durante la gestación y el puerperio precoz es el de la estimulación con ACTH. En casos leves es posible diferir la cirugía hasta después de la resolución del parto. Existen alternativas farmacológicas para el hipercortisolismo durante el embarazo que serían seguras tanto para la madre como para el feto.

\section{REFERENCIAS}

1. Lindsay JR, Jonklaas J, Oldfield EH, Nieman LK. Cushing's syndrome during pregnancy: experience and review of the literature. J Clin Endocrinol Metab 2005;90:3077-83.

2. Doshi S, Bhat A, Lim Kb. Cushing's syndrome in pregnancy. J Obstet Gynaecol 2003;23:5:568-9.

3. Nieman LK, Biller BM, Findling JW, Newell-Price J, Savage MO, Stewart PM, Montori VM. The diagnosis of Cushing's Syndrome: An Endocrine Society Clinical Practice Guideline. J Clin Endocrinol Metab 2008;93:1526-40.

4. Aron DC, Schnall AM, Sheeler LR. Cushing's syndrome and pregnancy. Am J Obst Gynecol 1990;162:4724.

5. Polli N, Pecori Giraldi F, Cavagnini F. Cushing's syndrome in pregnancy. J Endocrinol Invest 2003;26:1045-50.

6. Bevan JS, Gough MH, Gillmer MD, et al. Cushing's syndrome in pregnancy: the timing of definitive treatment. Clin Endocrinol (Oxf) 1987;27: 225-33.

7. Lindsay JR, Nieman LK. The hypothalamic-pituitaryadrenal axis in pregnancy: Challenges in disease detection and treatment. Endocr Rev 2005;26:775-99.

8. Marek B, Krysiak R, Okopień B. Adrenal disorders in pregnancy. Endocrinol Pol 2009;60:331-42.

9. Kreines K, Devaux WD. Neonatal adrenal insufficiency associated with maternal Cushing's syndrome. Pediatrics 1971;47:516-9.

10. Cousins L, Rigg L, Hollingsworth D, et al. Qualitative and quantitative assessment of the circadian rhythm of cortisol in pregnancy. Am J Obstet Gynecol 1983;145:411-6.

11. Bednarek-Tupikowska G, Kubicka E, Sicińska-Werner $\mathrm{T}$, et al. A case of Cushing's syndrome in pregnancy. Endokrynol Pol 2011;62:181-5.

12. Aishima M, Tanaka M, Haraoka M, et al. Retroperitoneal laparoscopic adrenalectomy in a pregnant woman with Cushing's syndrome. J Urol 2000;164:770-1. 
13. Connell JM, Cordiner J, Davies DL, et al. Pregnancy complicated by Cushing's syndrome: potential hazard of metyrapone therapy. Case report. Br J Obstet Gynaecol 1985;92:1192-5.

14. Kasperlik-Zaluska AA, Szczupacka I, Leszczynska-Bystrzanowska J, et al. Pregnancy-dependent Cushing's syndrome in three pregnancies. Br J Obstet Gynaecol 2000;107: 810-2.

15. Close CF, Mann MC, Watts JF, et al. ACTH-independent Cushing's syndrome in pregnancy with spontaneous resolution after delivery; control of the hypercortisolism with metyrapone. Clin Endocrinol (Oxf) 1993;39:375-9.
16. Amado JA, Pesquera C, Gonzalez EM, et al. Successful treatment with ketoconazole of Cushing's syndrome in pregnancy. Postgrad Med J 1990;66:221-3.

17. Berwaerts J, Verhelst J, Mahler C, et al. Cushing's syndrome in pregnancy treated by ketoconazole: case report and review of the literature. Gynecol Endocrinol 1999;13:175-82.

18. Heel RC, Brogden RN, Carmine A, et al. Ketoconazole: a review of its therapeutic efficacy in superficial and systemic fungal infections. Drugs 1982;23:1-23.

19. Hanson TJ, Ballonoff LB, Northcutt RC. Aminoglutethimide and pregnancy. JAMA 1974;230:963-64. 